Results For three, wheelchair-dependent patients with FND and FEVD admitted to The Wolfson Neuro-rehabilitation Centre for 12-24 week inpatient treatment, our approach resulted in two walking independently and one with supervision. Care needs were reduced and wheelchair dependence was eliminated. Patients reported improvement in independence and quality of life with one patient returning to parttime employment as a PA (See Table 1).

Conclusions With selective psychological and medical screening, invasive treatment for FEVD in FND patients delivered through a careful, stepwise treatment pathway produced excellent results for this subgroup of patients. Though such interventions are usually avoided for patients with FND, there may be a subgroup of patients for whom they remain useful as a treatment adjunct in order to maximise rehabilitation and functional outcomes.

\section{PREDICTION OF EMERGING AMYLOID AND TAU PATHOLOGY IN COGNITIVELY HEALTHY INDIVIDUALS: A LONGITUDINAL CSF STUDY}

${ }^{1}$ Ivan Koychev*, ${ }^{1}$ Nemanja Vaci, ${ }^{2}$ Murat Bilgel, ${ }^{3}$ Graciela Terrera Muniz, ${ }^{1}$ John Gallacher, ${ }^{2}$ Susan Resnick, ${ }^{4}$ Marilyn Albert. ${ }^{1}$ Department of Psychiatry, Oxford, UK; ${ }^{2}$ Laboratory of Behavioral Neuroscience, Intramural Research Program, National Institute on Aging, Baltimore, MD; ${ }^{3}$ Centre for Dementia Prevention, The University of Edinburgh; ${ }^{4}$ Department of Neurology, Johns Hopkins School of Medicine, Baltimore, MD

\subsection{6/jnnp-2019-BNPA.31}

Objectives/aims The importance of identifying emerging rather than widespread amyloid pathology is highlighted by evidence showing that rapid amyloid accumulation associates with cortical atrophy, cognitive deficits and tau deposition even where individuals are in the 'amyloid negative' range. The study aimed to test the hypothesis that among cognitively healthy individuals distinct groups exist in terms of amyloid and phosphorylated tau accumulation rates. We also hypothesised that membership to the faster accumulator group can be predicted (using age, genetics, cognition and hippocampal volume). Finally we aimed to identify time points of significant increase in the rate of $\mathrm{AD}$ protein accumulation and the associated predictors.

Methods The analysis reports data from 263 individuals from the BIOCARD NIH-funded study who had a mean of 2.23 cerebrospinal fluid (CSF) measurements since the study began in 1995. We used latent class mixed-effect models to identify distinct classes in terms of amyloid and p-tau accumulation rates. To investigate non-linear changes of AD-related CSF biomarkers we used generalised additive modelling. For both analyses demographic, genetic (APOE4 genotype) as well as repeated cognitive and structural MRI data were explored as predictors.

Results For both amyloid and p-tau latent class models we confirmed the existence of two separate classes: accumulators and non-accumulators. The accumulator and non-accumulator groups differed significantly in terms of baseline AD protein levels (mean age 60 at study inclusion) and slope. APOE4 homozygosity and cognitive reserve predicted amyloid class membership while APOE4 genotype only predicted p-tau class. The non-linear models revealed significant increase in the rate of amyloid and p-tau accumulation at ages 54 and age 55 respectively. APOE4 carrier status associated with earlier age of rapid accumulation start (ages 53 and 50 for amyloid p-tau respectively). Higher hippocampal volume but not cognitive ability was protective for amyloid accumulation. Neither hippocampal volume nor cognition were significant in the p-tau non-linear models.

Conclusions The current analysis demonstrates the existence of distinct classes of amyloid and p-Tau accumulators. Predictors for class membership were identified but the overall accuracy of the models was modest highlighting the need for biomarkers that are sensitive to early disease phenotypes. That amyloid and p-tau concentrations remain largely static until the mid-50s with a simultaneous sharp increase in accumulation thereafter also suggests that prediction of these points of inflection can be valuable in targeting specific time periods in disease modification trials.

\section{EMOTIONAL LABILITY IN HIPPOCAMPAL ATROPHY DUE TO AUTOIMMUNE LIMBIC ENCEPHALITIS}

${ }^{1}$ Georgios PD Argyropoulos, ${ }^{1,2}$ Lauren Moore, ${ }^{1,3}$ Clare Loane, ${ }^{1}$ Adriana Roca-Fernandez, ${ }^{1,4}$ Carmen Lage-Martinez, ${ }^{1}$ Christopher R Butler. ${ }^{1}$ University of Oxford; ${ }^{2}$ University of Bath: ${ }^{3}$ University College London; ${ }^{4}$ University Hospital Marqués de Valdecilla

\subsection{6/jnnp-2019-BNPA.32}

Objective/Aims Autoimmune limbic encephalitis (LE) is commonly associated with cognitive and psychiatric disturbances at the acute stage of the disease, and with residual episodic memory impairment. While behavioural and psychiatric symptoms generally dissipate post-acutely, very little is known about the profile of persistent neuropsychiatric symptoms. In particular, emotional lability represents an elusive entity that may be misdiagnosed as a manifestation of comorbid mood or personality change and can have disabling consequences, due to the stigma attached to the loss of emotional control. We aimed to assess the post-acute profile of emotional lability and its neuroanatomical correlates in LE.

Methods We analysed acute neuroradiological reports, clinical notes, scores on post-acute neuropsychological tests and selfadministered questionnaires on mood, emotion, and affect (including the Centre for Neurologic Study-Lability Scale; CNS-LS), along with structural MRI and resting-state fMRI datasets in relation to emotional lability in a large cohort of patients $(n=36)$ that had received a neurological diagnosis of LE, presented with focal hippocampal structural abnormalities in the acute phase, and post-acute hippocampal atrophy and thalamic volume reduction.

Results Emotional lability was present in $50 \%$ of the patients. It was associated with increased tearfulness compared with non-labile patients and healthy controls, whereas no patient presented with labile laughter (CNS-LS). Patients with emotional lability $(n=18)$ did not differ from those without $(n=18)$ in any demographic or clinical details in their acute or post-acute presentation (autoantibodies, immunosuppressive therapy, seizures, antidepressant medication, age at or delay from symptom onset), or in residual depression, anxiety, impulsiveness, memory impairment, or executive dysfunction, or in hippocampal and thalamic volumes. Instead, the presence and extent of emotional lability across patients was associated with reduced resting-state hemodynamic activity in and hippocampal functional connectivity with regions in the inferior and superior parietal lobules.

Conclusions We present the first investigation of persistent affective dysregulation in LE. Emotional lability is common following LE, but is not a manifestation of depression, anxiety, impulsiveness, or executive dysfunction. The type of 
emotional lability seen in LE is semiologically distinct from pseudobulbar affect observed in other neurological diseases. While LE is characterised by focal hippocampal atrophy, functional abnormalities in regions interacting with the hippocampus may provide a more parsimonious explanation of emotional lability than the volume of medial temporal lobe structures. Functional abnormalities in parietal regions supporting perspective taking and social-affective processing may compromise patients' emotion regulation.

\section{$33 \quad$ FIRST EPISODE PSYCHOSIS IN A PATIENT WITH EXTENSIVE LEUKOENCEPHALOPATHY DUE TO 3-METHYLGLUTACONIC ACIDURIA TYPE 4}

${ }^{1}$ Rebecca Charles*, ${ }^{2}$ Baskaran Sridharan. ${ }^{1}$ Foundation year 1 Doctor, Combined Healthcare NHS Trust; ${ }^{2}$ Consultant Neuropsychiatrist, Combined Healthcare NHS Trust

\subsection{6/jnnp-2019-BNPA.33}

Objectives/Aims This aim of case report is to discuss the clinical conundrum and diagnostic challenges in a young patient presenting with First Episode Psychosis. Investigations revealed Extensive Leukoencephalopathy due to a rare metabolic disorder- 3-methyloglutaconic aciduria (3-MGA) type IV. Several studies have shown that 3-MGA type IV can present with psychosis, epilepsy or depression as part of the spectrum of symptoms. The role of Organic Brain condition in the onset of first episode psychosis in this patient is discussed in this report.

Methods A 23-year-old female presented with insidious onset of paranoid delusions and auditory hallucinations over an 18 month period on a background of a diagnosis of 3-methylgutaconic aciduria type IV confirmed on urine testing. On admission under Section 2 of the Mental Health Act, she expressed little spontaneous speech and echolalia. She was flat in affect. She appeared vacant in expression, stared inappropriately and was very self-isolating. She was responding to external stimuli. She lacked insight into her condition. Physical examination was unremarkable. An MRI brain scan was performed, and comparison made to scan done previously to demonstrate any interval change and to correlate changes if present to deterioration of clinical symptoms.

Results MRI scan showed extensive diffuse leukoencephalopathy. Comparison to MRI scan done 6 years previously did not show any change or progression to the white matter lesions. An EEG showed a mild degree of general cerebral dysfunction with no interictal epileptiform activity. There was no correlation found between her clinical symptoms of acute onset psychosis and her diagnosis of 3-MGA Type IV. She was commenced on Aripiprazole and her presentation improved significantly. Both Auditory hallucinations and Paranoid delusions improved considerably, with moderate improvement in mood, affect and apathy. Some Catatonic symptoms persisted but were less intense. She was given a diagnosis of Undifferentiated Schizophrenia under ICD 10, as she displayed features of Paranoid, Hebephrenic and Catatonic without clear predominance of particular subtype of Schizophrenia. She was discharged home with follow-up from Neuropsychiatry and community Mental health teams. She continues to be investigated for the genetic cause of 3-methylglucatonic aciduria Type 4.

Conclusions To conclude, although often metabolic disorders, including 3-methylglucaconic aciduria, can present with psychosis, it is prudent to establish a causative link in order to manage appropriately and effectively.

\section{THE FUTURE ROLE OF FMRI NEUROFEEDBACK IN DEPRESSION TREATMENT AND RESEARCH}

*Zahn R, Jaeckle T, Williams SCR, Barker G, Young AH, Basilio R, Moll J. Centre for Affective Disorders, Department of Psychological Medicine Institute of Psychiatry, Psychology and Neuroscience (IoPPN), King's College London

\subsection{6/jnnp-2019-BNPA.34}

Objectives/Aims FMRI-neurofeedback for major depressive disorder (MDD) is of great interest to clinicians and neuroscientists. Here, the aim was to review the current clinical trials evidence.

Methods We undertook a systematic literature search of fMRIneurofeedback trials in MDD, including our unpublished results.

Results fMRI-neurofeedback was effective in current MDD when reinforcing brain responses to positive pictures, ${ }^{1}$ but was not superior to a control neurofeedback intervention in a recent randomised controlled trial ${ }^{2}$ (RCT). Another RCT showed that reinforcing amygdala responses to positive autobiographical memories ${ }^{3}$ was superior versus a control neurofeedback intervention ${ }^{4}$. We have developed neurofeedback of self-blame-selective functional connectivity between right superior anterior temporal (AT) and subgenual frontal regions. In remitted MDD, we demonstrated that self-esteem can be increased using this approach in a double-blind RCT. In a recently completed RCT in early treatment-resistant MDD, the majority of patients responded to guilt-related AT-subgenual connectivity neurofeedback. Surprisingly, a self-guided matched psychological intervention tackling self-blame without neurofeedback showed comparable levels of response. Secondary analyses, however, showed that neurofeedback was superior for those patients without anxious distress features.

Conclusions This calls for longer-term studies to reproduce previous results and stratified trials to combine psychological and neurofeedback interventions. As a research tool, neurofeedback uncovers causal relationships between functions and anatomical subdivisions.

\section{REFERENCES}

1. D. E. Linden, et al., PloS one 2012;7:e38115.

2. D. M. A. Mehler, et al., Neuropsychopharmacology, (2018).

3. K. D. Young, et al., PloS one 2014;9:e88785.

4. K. D. Young, et al., Am. J. Psychiatry 2017;174:748-755.

\section{THE EPIDEMIOLOGY AND SYMPTOMOLOGY OF FUNCTIONAL STROKE MIMICS: A SYSTEMATIC REVIEW AND META-ANALYSIS}

${ }^{1}$ Abbeygail Jones, ${ }^{1}$ Nicola O'Connell, ${ }^{2}$ Anthony David. ${ }^{1}$ Institute of Psychiatry, Psychology and Neuroscience, King's College London; ${ }^{2}$ Institute of Mental Health, University College London

\subsection{6/jnnp-2019-BNPA.35}

Aims Reconfiguration of stroke services in England has emphasised fast diagnosis and treatment and subsequently, the proportion of stroke mimic patients entering stroke pathways has been highlighted. Stroke mimic patients may be 'medical mimics', with medical explanations for symptoms, e.g. syncope, seizure, but a proportion of presenting patients have a 